Molecular Physics

Vol. 00, No. 00, Month 2014, 1-28

\title{
Manuscript
}

\section{On the exact formulation of multi-configuration density-functional theory: electron density versus orbitals occupation}

\author{
Emmanuel Fromager* \\ Laboratoire de Chimie Quantique, Institut de Chimie, CNRS / Université de Strasbourg, \\ 4 rue Blaise Pascal, 67000 Strasbourg, France;
}

(Received 00 Month 200x; final version received 00 Month 200x)

\begin{abstract}
The exact formulation of multi-configuration density-functional theory (DFT) is discussed in this work. As an alternative to range-separated methods, where electron correlation effects are split in the coordinate space, the combination of Configuration Interaction methods with orbital occupation functionals is explored at the formal level through the separation of correlation effects in the orbital space. When applied to model Hamiltonians, this approach leads to an exact Site-Occupation Embedding Theory (SOET). An adiabatic connection expression is derived for the complementary bath functional and a comparison with Density Matrix Embedding Theory (DMET) is made. Illustrative results are given for the simple two-site Hubbard model. SOET is then applied to a quantum chemical Hamiltonian, thus leading to an exact Complete Active Space Site-Occupation Functional Theory (CASSOFT) where active electrons are correlated explicitly within the CAS and the remaining contributions to the correlation energy are described with an orbital occupation functional. The computational implementation of SOET and CASSOFT as well as the development of approximate functionals are left for future work.
\end{abstract}

Keywords: Density-Functional Theory, Range Separation, Multi-Configurational Methods, Site-Occupation Embedding Theory, Strongly Correlated Systems.

\section{Introduction}

The description of strong electron correlation effects is still nowadays a challenging problem for both density-functional theory (DFT) and wavefunction theory (WFT) communities. Even though Kohn-Sham DFT (KS-DFT) is in principle exact, standard approximate exchange-correlation functionals usually do not enable an adequate description of multi-configurational systems. On the other hand, the "Gold standard" single-reference Coupled Cluster (CC) method should also be able to model near-degeneracies but high-order excitations would then be necessary. In practice multi-reference (MR) perturbation theories such as the second order Complete Active Space Perturbation Theory (CASPT2) [1, 2] or the $N$-Electron Valence state Perturbation Theory (NEVPT2) [3, 4] are usually employed. Despite their success, these methods may suffer from the perturbative description of the short-range dynamical correlation which is sometimes not accurate enough. For these reasons multi-configurational extensions of both DFT and CC have been investigated for many years and despite significant efforts and

*Corresponding author. Email: fromagere@unistra.fr 
encouraging results, it is still unclear how the incorporation of a Complete Active Space Self-Consistent Field (CASSCF) into DFT and CC should be performed. A recent review article by Bartlett and coworkers [5] gives an extensive picture of the developments in MRCC. We shall focus here on DFT and restrict ourselves to the description of the ground state.

The rigorous combination of CASSCF with DFT is already difficult at the formal level due to the infamous double counting problem. Specific functionals must indeed be developed for complementing the CASSCF energy that already contains some correlation effects [6-10]. The latter are usually referred to as static correlation effects. Only the complementary correlation, known as dynamical correlation, should therefore be assigned to the density functional. Turning such a scheme into a practical computational method is not an easy task since approximations used for standard functionals cannot be applied straightfowardly in this context. One way to overcome the double counting problem consists in separating correlation effects in the coordinate space, as initially proposed by Savin [11]. The resulting rangeseparated DFT methods combine rigorously long-range WFT with short-range DFT. Even though range separation allows for a multi-configurational description of the electron density, it cannot completely isolate static correlation from dynamical correlation simply because the former is usually not a purely long-range effect, even in a dissociated molecule. This point will be discussed further in the following.

Let us mention that Savin earlier combined Configuration Interaction (CI) with DFT by separating correlation effects in the natural orbital space [12. Orbitals with occupation numbers larger than a given threshold $\nu$ were correlated explicitly, at the CI level, while the remaining correlation energy was described with a complementary $\nu$-dependent density functional. More recently, Gutlé and Savin [13] proposed alternative CI-DFT schemes where the correlation energy is still split in the orbital space but through the introduction of gap shift or cutoff parameters. In all these hybrid CI-DFT schemes, a CI energy is complemented by a functional of the electron density. In this work we propose to revisit at the formal level the separation of correlation effects in the orbital space. As a major difference with the approaches discussed previously, orbitals occupation will be used as basic variable rather than the electron density. The idea originates from condensed matter physics where strongly correlated electrons are usually described with model Hamiltonians (such as the Hubbard Hamiltonian) rather than the true physical one [14]. As discussed further in the following, adapting such an approach to quantum chemistry is appealing since static correlation is usually defined in the orbital space. Molecular orbitals can then be viewed as sites with an occupation to be determined. As shown in this work, a WFT description of static correlation can be combined rigorously with an orbital-occupation functional modeling of dynamical correlation. A potential drawback of such a scheme lies in the fact that, in contrast to range-separated DFT, the complementary functional loses its universality since it depends on the molecular orbitals. The theory is derived for a particular choice of orbitals, namely those obtained by diagonalizing the non-interacting Hamiltonian (kinetic and nuclear potential energy operators only are considered). Its generalization is left for future work.

The paper is organized as follows: After a short introduction to the double counting problem in multi-configuration DFT (Sec. 2), the exact multi-determinant extension of standard DFT based on range separation is briefly reviewed (Sec. 3.1) and its application to the dissociated $\mathrm{H}_{2}$ molecule is discussed in Sec. 3.2. We then 
leave the framework of DFT by using orbitals occupation as basic variable rather than the electron density. This approach is first introduced for model Hamiltonians such as the Hubbard Hamiltonian (Sec. 4). By isolating an impurity site and separating its on-site repulsion from the interactions on the remaining sites (referred to as the bath), an exact Site-Occupation Embedding Theory (SOET) is derived and compared with the Density Matrix Embedding Theory (DMET) of Knizia and Chan [15]. Illustrative results are then given for the simple two-site Hubbard model in Sec. 5. Finally, we show in Sec. 6 that an exact combination of CI with orbital occupation functional theory is obtained when applying SOET to a quantum chemical Hamiltonian. Conclusions and perspectives are given in Sec. 7.

\section{The double counting problem}

In WFT the exact ground-state energy of an electronic system can be obtained variationally as follows,

$$
E=\min _{\Psi}\left\langle\Psi\left|\hat{T}+\hat{V}_{\mathrm{ne}}+\hat{W}_{\mathrm{ee}}\right| \Psi\right\rangle
$$

where $\hat{T}$ is the kinetic energy operator, $\hat{W}_{\text {ee }}$ denotes the two-electron repulsion operator and $\hat{V}_{\text {ne }}=\int \mathrm{d} \mathbf{r} v_{\text {ne }}(\mathbf{r}) \hat{n}(\mathbf{r})$ is the nuclear potential operator. In a regular CASSCF calculation, the minimization in Eq. (1) is restricted to linear combinations of Slater determinants that belong to a given Complete Active Space (CAS). The orbitals are also optimized variationally. The CAS is obtained by distributing a given number of active electrons in selected active orbitals that become consequently partially occupied. The selection of active electrons and orbitals is usually based on chemical intuition. Doubly occupied orbitals are referred to as inactive orbitals. Note that the latter are not frozen in a CASSCF calculation. The remaining orbitals, that are not occupied, are the virtuals. For convenience we will denote $\mathcal{S}^{M}$ the space of trial CASSCF wavefunctions. The superscript $M$ specifies all the restrictions in the minimization (number of active electrons and active orbitals for example). The CASSCF energy can therefore be written as

$$
E_{\mathrm{CASSCF}}=\min _{\Psi \in \mathcal{S}^{M}}\left\langle\Psi\left|\hat{T}+\hat{V}_{\mathrm{ne}}+\hat{W}_{\mathrm{ee}}\right| \Psi\right\rangle
$$

Note that, in practice, a trial CASSCF wavefunction $\Psi$ will be parametrized in second quantization as follows [16],

$$
\forall \Psi \in \mathcal{S}^{M},|\Psi\rangle=e^{-\hat{\kappa}}\left(\sum_{I \in \mathrm{CAS}} C_{I}\left|\operatorname{det}_{I}\right\rangle\right),
$$

where $\left\{\operatorname{det}_{I}\right\}_{I \in \mathrm{CAS}}$ is the basis of determinants for the CAS. The singlet excitation operator $\hat{\kappa}$ that allows for orbital rotations is defined as

$$
\hat{\kappa}=\sum_{p>q, \sigma=\uparrow, \downarrow} \kappa_{p q}\left(\hat{a}_{p \sigma}^{\dagger} \hat{a}_{q \sigma}-\hat{a}_{q \sigma}^{\dagger} \hat{a}_{p \sigma}\right)
$$

where $p$ and $q$ can be inactive, active or virtual orbitals. Consequently, the minimization in Eq. (2) is performed over both $\left\{\kappa_{p q}\right\}_{p>q}$ and $\left\{C_{I}\right\}_{I \in \text { CAS }}$ parameters. 
The energy difference $E-E_{\mathrm{CASSCF}}$ is usually referred to as dynamical correlation energy. The latter is routinely described within multi-reference perturbation theories such as CASPT2 or NEVPT2. A combined CASSCF-DFT approach should ideally be exact and variational. Dynamical correlation would be described with a density functional $\bar{E}_{\mathrm{c}}^{\mathrm{dyn}}[n]$ that would complement the CASSCF energy. This can be formulated rigorously when considering the universal Levy-Lieb functional [17,

$$
F[n]=\min _{\Psi \rightarrow n}\left\langle\Psi\left|\hat{T}+\hat{W}_{\mathrm{ee}}\right| \Psi\right\rangle
$$

where the minimization is performed over all wavefunctions with density $n$, and the following partitioning [7],

$$
F[n]=F^{M}[n]+\bar{E}_{\mathrm{c}}^{\mathrm{dyn}}[n] .
$$

The LL functional associated with the CASSCF space $\mathcal{S}^{M}$ is defined as

$$
F^{M}[n]=\min _{\Psi \in \mathcal{S}^{M} \rightarrow n}\left\langle\Psi\left|\hat{T}+\hat{W}_{\mathrm{ee}}\right| \Psi\right\rangle,
$$

where the minimization is restricted to CASSCF wavefunctions in $\mathcal{S}^{M}$ with density $n$. According to the Hohenberg-Kohn (HK) theorem [18, the exact ground-state energy can be obtained variationally as follows,

$$
E=\min _{n}\left\{F[n]+\left(v_{\text {ne }} \mid n\right)\right\}
$$

where the minimization is performed over electron densities $n(\mathbf{r})$ that integrate to a fixed number $N$ of electrons. The notation $(v \mid n)=\int \mathrm{d} \mathbf{r} v(\mathbf{r}) n(\mathbf{r})$ has been used. Combining Eq. (6) with Eq. (8) leads to

$$
E=\min _{n}\left\{F^{M}[n]+\bar{E}_{\mathrm{c}}^{\mathrm{dyn}}[n]+\left(v_{\mathrm{ne}} \mid n\right)\right\} .
$$

Consequently, for any trial CASSCF wavefunction $\Psi$ in $\mathcal{S}^{M}$,

$$
\begin{aligned}
\left\langle\Psi\left|\hat{T}+\hat{V}_{\mathrm{ne}}+\hat{W}_{\mathrm{ee}}\right| \Psi\right\rangle+\bar{E}_{\mathrm{c}}^{\mathrm{dyn}}\left[n_{\Psi}\right] & \geq F^{M}\left[n_{\Psi}\right]+\bar{E}_{\mathrm{c}}^{\mathrm{dyn}}\left[n_{\Psi}\right]+\left(v_{\mathrm{ne}} \mid n_{\Psi}\right) \\
& \geq E
\end{aligned}
$$

where $n_{\Psi}(\mathbf{r})=\langle\Psi|\hat{n}(\mathbf{r})| \Psi\rangle$ is the electron density obtained from the trial CASSCF wavefunction $\Psi$. Thus we conclude that, provided that the exact ground-state density can be reproduced by a CASSCF wavefunction, the exact ground-state energy can be written as

$$
E=\min _{\Psi \in \mathcal{S}^{M}}\left\{\left\langle\Psi\left|\hat{T}+\hat{V}_{\mathrm{ne}}+\hat{W}_{\mathrm{ee}}\right| \Psi\right\rangle+\bar{E}_{\mathrm{c}}^{\mathrm{dyn}}\left[n_{\Psi}\right]\right\} .
$$

In order to turn Eq. 11 into a practical computational method, approximate complementary correlation functionals should be developed. Obviously standard correlation functionals cannot be used otherwise correlation effects would be double counted. This double counting problem arises also when the size of the CAS varies. In the exact theory, the functional should vary with the CAS so that correlation effects are transferred from the CASSCF to the DFT part of the energy and the total energy remains constant and equal to the exact ground-state energy. 
Deriving CAS-dependent density functionals is a complicated task simply because the electron density is defined in the coordinate space while a CAS is defined in the orbital space. In order to rigorously overcome such difficulties, Savin [11] proposed to separate correlation effects in the coordinate space, thus leading to the so-called range-separated DFT methods. This approach is briefly reviewed in Sec. 3. We then propose an alternative approach where correlation effects are separated in the orbital space, like in a regular CASSCF calculation. In this context, the orbitals occupation will be used as basic variable rather than the electron density.

\section{Multi-configuration range-separated DFT}

\section{1. $\quad$ Separating correlations in coordinate space}

In the standard KS-DFT scheme [19, the universal LL functional in Eq. (5) is decomposed into the non-interacting kinetic energy functional $T_{\mathrm{S}}[n]=\min _{\Psi \rightarrow n}\langle\Psi|\hat{T}| \Psi\rangle$ and the Hartree-exchange-correlation (Hxc) energy functional,

$$
F[n]=T_{\mathrm{s}}[n]+E_{\mathrm{Hxc}}[n] .
$$

Consequently, the HK variational principle can be reformulated as follows,

$$
E=\min _{\Psi}\left\{\left\langle\Psi\left|\hat{T}+\hat{V}_{\mathrm{ne}}\right| \Psi\right\rangle+E_{\mathrm{Hxc}}\left[n_{\Psi}\right]\right\},
$$

where the minimizing KS determinant $\Phi^{\mathrm{KS}}$ fulfils the self-consistent equation

$$
\left(\hat{T}+\hat{V}_{\mathrm{ne}}+\int \mathrm{d} \mathbf{r} \frac{\delta E_{\mathrm{Hxc}}}{\delta n(\mathbf{r})}\left[n_{\Phi^{\mathrm{KS}}}\right] \hat{n}(\mathbf{r})\right)\left|\Phi^{\mathrm{KS}}\right\rangle=\mathcal{E}^{\mathrm{KS}}\left|\Phi^{\mathrm{KS}}\right\rangle .
$$

Therefore, within KS-DFT, the two-electron interaction is fully described by a density functional. As originally shown by Savin [11, it is in fact possible to describe only a part of the two-electron repulsion within DFT and leave the remaining part to WFT. This is achieved by separating the interaction in the coordinate space into two complementary contributions. The range separation based on the error function,

$$
\begin{aligned}
w_{\mathrm{ee}}\left(r_{12}\right) & =w_{\mathrm{ee}}^{\mathrm{lr}, \mu}\left(r_{12}\right)+w_{\mathrm{ee}}^{\mathrm{sr}, \mu}\left(r_{12}\right), \\
w_{\mathrm{ee}}^{\mathrm{lr}, \mu}\left(r_{12}\right) & =\operatorname{erf}\left(\mu r_{12}\right) / r_{12}
\end{aligned}
$$

has for example been used extensively in the last decade (see Ref. [20] and the references therein), but any separation like the simpler linear one [21] can be considered. Range separation, that is controlled by the $\mu$ parameter, is appealing as it enables to isolate the Coulomb hole and assign it to a density functional while long-range correlation can be described in WFT. This hybrid range-separated WFT-DFT approach can be derived rigorously from the alternative partitioning of the LL functional,

$$
F[n]=F^{\mathrm{lr}, \mu}[n]+\bar{E}_{\mathrm{Hxc}}^{\mathrm{sr}, \mu}[n],
$$


where the long-range LL functional equals

$$
F^{\mathrm{lr}, \mu}[n]=\min _{\Psi \rightarrow n}\left\langle\Psi\left|\hat{T}+\hat{W}_{\mathrm{ee}}^{\mathrm{lr}, \mu}\right| \Psi\right\rangle,
$$

and $\bar{E}_{\mathrm{Hxc}}^{\mathrm{sr}, \mu}[n]$ is the complementary $\mu$-dependent short-range Hxc density functional. Let us stress that, for any $\mu$ value, this functional is universal since it depends on the electron density only. Combining Eq. (16) with Eq. (8) leads to the exact ground-state energy expression

$$
E=\min _{\Psi}\left\{\left\langle\Psi\left|\hat{T}+\hat{W}_{\mathrm{ee}}^{\mathrm{lr}, \mu}+\hat{V}_{\mathrm{ne}}\right| \Psi\right\rangle+\bar{E}_{\mathrm{Hxc}}^{\mathrm{sr}, \mu}\left[n_{\Psi}\right]\right\},
$$

where the minimizing wavefunction $\Psi^{\mu}$ fulfils the self-consistent equation

$$
\begin{aligned}
& \hat{H}^{\mu}\left|\Psi^{\mu}\right\rangle=\mathcal{E}^{\mu}\left|\Psi^{\mu}\right\rangle, \\
& \hat{H}^{\mu}=\hat{T}+\hat{W}_{\mathrm{ee}}^{\mathrm{lr}, \mu}+\hat{V}_{\mathrm{ne}}+\int \mathrm{d} \mathbf{r} \frac{\delta \bar{E}_{\mathrm{Hxc}}^{\mathrm{sr}, \mu}}{\delta n(\mathbf{r})}\left[n_{\Psi^{\mu}}\right] \hat{n}(\mathbf{r}) .
\end{aligned}
$$

Note that KS-DFT and pure WFT are recovered when $\mu=0$ and $\mu \rightarrow+\infty$, respectively.

Since the long-range interaction is now described explicitly, the auxiliary wavefunction $\Psi^{\mu}$, that has exactly the same density as the physical system, is multi-determinantal. CASSCF can therefore be applied in this context in conjunction with local or semi-local short-range functionals [22, 23. Even though encouraging results were obtained with such a range-separated CASDFT scheme, better functionals are still needed for the method to be reliable [20]. As discussed further in Sec. 3.2, one major problem is that static correlation is not a purely long-range correlation effect.

Returning to the exact theory, an adiabatic connection (AC) [24 29] expression for the short-range functional can be obtained from the auxiliary equations,

$$
\left(\hat{T}+\hat{W}_{\mathrm{ee}}^{\mathrm{lr}, \nu}+\int \mathrm{d} \mathbf{r} v^{\nu}(\mathbf{r}) \hat{n}(\mathbf{r})\right)\left|\Psi^{\nu}\right\rangle=\mathcal{E}^{\nu}\left|\Psi^{\nu}\right\rangle,
$$

and the density constraint $n_{\Psi^{\nu}}(\mathbf{r})=n(\mathbf{r})$ for $0 \leq \nu<+\infty$. Indeed, according to Eqs. (16) and (17),

$$
\begin{aligned}
\bar{E}_{\mathrm{Hxc}}^{\mathrm{sr}, \mu}[n] & =F[n]-F^{\mathrm{lr}, \mu}[n] \\
& =\int_{\mu}^{+\infty} \frac{\mathrm{d} \mathcal{E}^{\nu}}{\mathrm{d} \nu} \mathrm{d} \nu+\left(v^{\mu}-v^{+\infty} \mid n\right),
\end{aligned}
$$

thus leading, according to the Hellmann-Feynman theorem, to

$$
\bar{E}_{\mathrm{Hxc}}^{\mathrm{sr}, \mu}[n]=\int_{\mu}^{+\infty}\left\langle\Psi^{\nu}\left|\partial \hat{W}_{\mathrm{ee}}^{\mathrm{lr}, \nu} / \partial \nu\right| \Psi^{\nu}\right\rangle
$$

We obtain for $\mu=0$ a range-separated AC expression for the conventional Hxc 
functional,

$$
E_{\mathrm{Hxc}}[n]=\int_{0}^{+\infty}\left\langle\Psi^{\nu}\left|\partial \hat{W}_{\mathrm{ee}}^{\mathrm{lr}, \nu} / \partial \nu\right| \Psi^{\nu}\right\rangle
$$

It is readily seen from Eqs. (22) and (23) that conventional functionals cannot be used straightfowardly in multi-determinant range-separated DFT otherwise there would be double counting of long-range correlation effects through the WFT treatment. By connecting the non-interacting $\mathrm{KS}$ system $(\nu=0)$ to the long-range interacting one $(\nu=\mu)$ as follows,

$$
\begin{aligned}
\int_{0}^{\mu}\left\langle\Psi^{\nu}\left|\partial \hat{W}_{\mathrm{ee}}^{\mathrm{lr}, \nu} / \partial \nu\right| \Psi^{\nu}\right\rangle & =\int_{0}^{\mu} \frac{\mathrm{d} \mathcal{E}^{\nu}}{\mathrm{d} \nu} \mathrm{d} \nu+\left(v^{0}-v^{\mu} \mid n\right)=F^{\mathrm{lr}, \mu}[n]-T_{\mathrm{s}}[n] \\
& =E_{\mathrm{Hxc}}^{\mathrm{lr}, \mu}[n],
\end{aligned}
$$

where $E_{\mathrm{Hxc}}^{\mathrm{lr}, \mu}[n]$ is the purely long-range Hxc functional, we obtain from Eqs. 22 and (23) the following expression,

$$
\bar{E}_{\mathrm{Hxc}}^{\mathrm{sr}, \mu}[n]=E_{\mathrm{Hxc}}[n]-E_{\mathrm{Hxc}}^{\mathrm{lr}, \mu}[n],
$$

that has been used by Toulouse et al. [30] for developing approximate local and semi-local short-range functionals. We should stress that all the formalism briefly reviewed in this section is in fact general and can be applied in a different context, for example in DFT for model Hamiltonians, as proposed in Sec. 4.2.

\subsection{Left-right correlation and range separation}

We consider in this section the $\mathrm{H}_{2}$ molecule in a Slater minimal basis consisting of the $1 s_{A}$ and $1 s_{B}$ atomic orbitals localized on the left and right hydrogen atoms, respectively [21, 31]. The basis functions are identical with $\zeta=1$. For large bond distances the bonding and anti-bonding molecular orbitals are equal to $1 \sigma_{g}=$ $\frac{1}{\sqrt{2}}\left(1 s_{A}+1 s_{B}\right)$ and $1 \sigma_{u}=\frac{1}{\sqrt{2}}\left(1 s_{A}-1 s_{B}\right)$, respectively. The ground state will then be written in the basis the two Slater determinants $1 \sigma_{g}^{2}$ and $1 \sigma_{u}^{2}$. Since the latter differ by a double excitation, they are not coupled by one-electron operators. Therefore, when approaching the dissociation limit, the matrix representation of the auxiliary long-range Hamiltonian in Eq. (19) reduces to

$$
\left[\hat{H}^{\mu}\right]=\left[\begin{array}{ll}
E^{\mu} & K^{\mu} \\
K^{\mu} & E^{\mu}
\end{array}\right]
$$

where diagonal elements are identical since atomic orbitals do not overlap, and the coupling term equals

$$
\begin{aligned}
K^{\mu} & =\left\langle 1 \sigma_{g}^{2}\left|\hat{W}_{\mathrm{ee}}^{\mathrm{lr}, \mu}\right| 1 \sigma_{u}^{2}\right\rangle=\left\langle 1 \sigma_{u} 1 \sigma_{u}\left|w_{\mathrm{ee}}^{\mathrm{lr}, \mu}\left(r_{12}\right)\right| 1 \sigma_{g} 1 \sigma_{g}\right\rangle \\
& =\frac{1}{2}\left\langle 1 s_{A} 1 s_{A}\left|w_{\mathrm{ee}}^{\mathrm{lr}, \mu}\left(r_{12}\right)\right| 1 s_{A} 1 s_{A}\right\rangle-\frac{1}{2}\left\langle 1 s_{A} 1 s_{B}\left|w_{\mathrm{ee}}^{\mathrm{lr}, \mu}\left(r_{12}\right)\right| 1 s_{A} 1 s_{B}\right\rangle .
\end{aligned}
$$

While the second long-range two-electron integral on the right-hand side of Eq. (27) reduces to the regular one $\left\langle 1 s_{A} 1 s_{B} \mid 1 s_{A} 1 s_{B}\right\rangle$ and becomes zero in the dissociation limit, the first term is an "on-site" integral computed with the long-range interaction. Thus we conclude that the coupling between $1 \sigma_{g}^{2}$ and $1 \sigma_{u}^{2}$ determinants is 
determined by the contribution at short range of the long-range interaction. In this case static correlation, that is also referred to as left-right correlation, can obviously not be interpreted as a purely long-range correlation effect. Nevertheless, if the error function is used, we see from its Taylor expansion for small $\mu r_{12}$,

$$
w_{\mathrm{ee}}^{\mathrm{lr}, \mu}\left(r_{12}\right)=\frac{2}{\sqrt{\pi}}\left(\mu-\frac{1}{3} \mu^{3} r_{12}^{2}+\ldots\right)
$$

that the coupling term can be expanded as follows

$$
K^{\mu}=\frac{\mu}{\sqrt{\pi}}+\ldots
$$

Consequently, as already pointed out by Gori-Giorgi and Savin [32], even an infinitesimal $\mu$ value ensures that $K^{\mu}$ is not strictly equal to zero, thus providing the correct multi-configurational description of the dissociated $\mathrm{H}_{2}$ molecule in the ground state:

$$
\left|\Psi^{\mu}\right\rangle=\frac{1}{\sqrt{2}}\left(\left|1 \sigma_{g}^{2}\right\rangle-\left|1 \sigma_{u}^{2}\right\rangle\right)
$$

This simple example illustrates how difficult it is to describe static correlation in the coordinate space, in contrast to short-range dynamical correlation that is connected with the Coulomb hole. We propose in the rest of this paper an alternative approach where correlations are separated in the orbital space. In order to overcome the double counting problem, the orbitals occupation will be used rather than

the electron density. For clarity, this approach will be introduced first for model Hamiltonians.

\section{DFT for model Hamiltonians}

As mentioned previously, it is convenient to work in the orbital space rather than the coordinate space when it comes to separate static and dynamical correlation effects. A change of paradigm is then necessary in order to avoid the double counting problem. The basic variable in DFT is the electron density $n(\mathbf{r})$ that is defined in the coordinate space. As we want to reformulate DFT in the orbital space, orbitals occupation $\left\{n_{i}\right\}_{i}$ seems to be the variable of choice. This is known in condensed matter physics as Site-Occupation Functional Theory (SOFT) [14, 33, 34]. The latter is nothing but the formulation of DFT for model Hamiltonians such as the Hubbard Hamiltonian. After a short introduction to the KS-SOFT scheme in Sec. 4.1, we will show in Sec. 4.2 how formal analogies with range-separated DFT can lead in this context to an exact embedding theory for model Hamiltonians. 


\subsection{SOFT and its $K S$ formulation}

Let us consider the Hubbard Hamiltonian with an external potential $v \equiv\left\{v_{i}\right\}_{i}$ :

$$
\begin{array}{r}
\hat{H}=\hat{\mathcal{T}}+\hat{U}+\sum_{i} v_{i} \hat{n}_{i}, \\
\hat{\mathcal{T}}=-t \sum_{i \neq j, \sigma} \hat{a}_{i \sigma}^{\dagger} \hat{a}_{j \sigma} \\
\hat{U}=U \sum_{i} \hat{n}_{i \uparrow} \hat{n}_{i \downarrow},
\end{array}
$$

where $t$ is the hopping integral, $U$ denotes the on-site two-electron repulsion, $\hat{n}_{i \sigma}=$ $\hat{a}_{i \sigma}^{\dagger} \hat{a}_{i \sigma}$ and $\sigma=\uparrow, \downarrow$. The site-occupation operator equals $\hat{n}_{i}=\hat{n}_{i \uparrow}+\hat{n}_{i \downarrow}$ and, for a given wavefunction $\Psi$, the occupation of site $i$ is defined as $n_{i}=\left\langle\Psi\left|\hat{n}_{i}\right| \Psi\right\rangle$. For simplicity we will consider a fixed number of electrons $N$ and only discuss ground-state properties. As shown by Gunnarsson and Schönhammer [33], the HK theorem can be adapted to the Hamiltonian in Eq. (31). There is indeed a oneto-one correspondence between the external potential $v$ and the ground-state sites occupancy $n \equiv\left\{n_{i}\right\}_{i}$. Consequently, for fixed $t$ and $U$ parameters, the exact groundstate energy can be obtained from the following variational principle,

$$
E(v)=\min _{n}\{F(n)+(v \mid n)\}
$$

where $(v \mid n)=\sum_{i} v_{i} n_{i}$. The analog of the universal HK functional is a function of the sites occupation that can be written within the LL constrained-search formalism as

$$
F(n)=\min _{\Psi \rightarrow n}\{\langle\Psi|\hat{\mathcal{T}}+\hat{U}| \Psi\rangle\}
$$

Since the HK theorem still holds when $U=0$, a KS formulation of SOFT (KSSOFT) is obtained from the following partitioning,

$$
F(n)=\mathcal{T}_{\mathrm{s}}(n)+E_{\mathrm{Hxc}}(n),
$$

where the non-interacting kinetic energy functional is defined in analogy with KSDFT as

$$
\mathcal{T}_{\mathrm{s}}(n)=\min _{\Psi \rightarrow n}\{\langle\Psi|\hat{\mathcal{T}}| \Psi\rangle\}=\left\langle\Psi^{\mathrm{KS}}(n)|\hat{\mathcal{T}}| \Psi^{\mathrm{KS}}(n)\right\rangle
$$

Note that, in this context, the non-interacting KS system is not described by sites that are strictly singly, doubly or not occupied,

$$
\begin{aligned}
& \left|\Psi^{\mathrm{KS}}(n)\right\rangle \neq \prod_{i, \sigma}\left(\hat{a}_{i \sigma}^{\dagger}\right)^{n_{i \sigma}}|\mathrm{vac}\rangle, \\
& n_{i \sigma}=0,1 \\
& \sum_{i, \sigma} n_{i \sigma}=N
\end{aligned}
$$

In other words $\Psi^{\mathrm{KS}}(n)$ is not a single Slater determinant in the basis of the site orbitals. Indeed, $\Psi^{\mathrm{KS}}(n)$ is the ground state of the non-interacting Hamiltonian 
$\hat{\mathcal{T}}+\sum_{i} v_{i}^{\mathrm{KS}}(n) \hat{n}_{i}$ where $\hat{\mathcal{T}}$ is non-local in the orbital space. Let us stress that there is no HK theorem without a non-local term like $\hat{\mathcal{T}}$ included into the Hamiltonian. For example, for two electrons, the two Hamiltonians that differ by more than a constant,

$$
\varepsilon_{1} \hat{n}_{1}+\varepsilon_{2} \hat{n}_{2}
$$

and

$$
\varepsilon_{1} \hat{n}_{1}+\left(\varepsilon_{2}+\delta\right) \hat{n}_{2}
$$

where $\varepsilon_{1}<\varepsilon_{2}$ and $\delta>\varepsilon_{1}-\varepsilon_{2}$, yield the same ground-state occupancies $n_{1}=2$ and $n_{2}=0$. This is an important difference with KS-DFT where the quantity to reproduce from singly or doubly occupied KS orbitals is the exact electron density $n(\mathbf{r})$. In KS-SOFT, the quantity to reproduce is the exact sites occupation $n$ that may be fractional due to the non-zero hopping integral $t$.

By analogy with KS-DFT, an exact AC expression for the Hxc site-occupation functional in Eq. (34) can be obtained from the following auxiliary equations,

$$
\left(\hat{\mathcal{T}}+\lambda \hat{U}+\sum_{i} v_{i}^{\lambda} \hat{n}_{i}\right)\left|\Psi^{\lambda}\right\rangle=\mathcal{E}^{\lambda}\left|\Psi^{\lambda}\right\rangle
$$

with the site-occupation constraint $n_{\Psi^{\lambda}}=n$ fulfilled for $0 \leq \lambda \leq 1$, thus leading to

$$
\begin{aligned}
E_{\mathrm{Hxc}}(n) & =\int_{0}^{1} \mathrm{~d} \lambda \frac{\mathrm{d} \mathcal{E}^{\lambda}}{\mathrm{d} \lambda}+\left(v^{0}-v^{1} \mid n\right) \\
& =\int_{0}^{1} \mathrm{~d} \lambda\left\langle\Psi^{\lambda}|\hat{U}| \Psi^{\lambda}\right\rangle,
\end{aligned}
$$

according to the Hellmann-Feynman theorem. Note that within the mean-field approximation and in the particular case of a singlet ground state, the Hxc functional is simplified as follows,

$$
E_{\mathrm{Hxc}}(n) \rightarrow U \sum_{i} n_{i \uparrow} n_{i \downarrow}=\frac{U}{4} \sum_{i} n_{i}^{2}
$$

The latter expression is usually referred to as the Hartree energy in condensed matter physics [14]. The exchange energy is then considered to be zero.

\subsection{Exact embedding within SOFT}

In the spirit of Dynamical Mean-Field Theory [35, 36] and DMET [15], we propose to isolate one site, referred to as impurity and for which correlation effects will be described explicitly, while the remaining sites (the bath) are treated within SOFT. Such an approach, that will be referred to as Site-Occupation Embedding Theory (SOET) in the following, is formally very similar to range-separated DFT. The main difference is that the interactions are separated here in the orbital space rather than in the coordinate space. For convenience the impurity and bath sites will be labelled as $i=0$ and $i>0$, respectively. By analogy with Eqs. (16) and 
(17), SOET can be derived from the following partitioning of the LL functional,

$$
F(n)=F^{\mathrm{imp}}(n)+\bar{E}_{\mathrm{Hxc}}^{\mathrm{bath}}(n),
$$

where the impurity LL functional equals

$$
F^{\mathrm{imp}}(n)=\min _{\Psi \rightarrow n}\left\{\left\langle\Psi\left|\hat{\mathcal{T}}+U \hat{n}_{0 \uparrow} \hat{n}_{0 \downarrow}\right| \Psi\right\rangle\right\}
$$

and $\bar{E}_{\mathrm{Hxc}}^{\mathrm{bath}}(n)$ denotes the complementary Hxc bath functional that describes the bath as well as the coupling between the bath and the impurity. Note that, in contrast to range-separated DFT, the separation of correlation effects is, in SOET, not controlled by a single continuous parameter. It rather relies on the selection of an impurity site. As discussed in the following more impurity sites can actually be considered.

We now return to the single-impurity case. Since, according to Eqs. (32), (42) and (43), any normalized trial wavefunction $\Psi$ fulfils

$$
\begin{aligned}
& \left\langle\Psi\left|\hat{\mathcal{T}}+U \hat{n}_{0 \uparrow} \hat{n}_{0 \downarrow}\right| \Psi\right\rangle+\bar{E}_{\mathrm{Hxc}}^{\text {bath }}\left(n_{\Psi}\right)+\left(v \mid n_{\Psi}\right) \\
& \geq F^{\mathrm{imp}}\left(n_{\Psi}\right)+\bar{E}_{\mathrm{Hxc}}^{\mathrm{bath}}\left(n_{\Psi}\right)+\left(v \mid n_{\Psi}\right) \geq E(v),
\end{aligned}
$$

the exact ground-state energy can be expressed as

$$
E(v)=\min _{\Psi}\left\{\left\langle\Psi\left|\hat{\mathcal{T}}+U \hat{n}_{0 \uparrow} \hat{n}_{0 \downarrow}\right| \Psi\right\rangle+\bar{E}_{\mathrm{Hxc}}^{\mathrm{bath}}\left(n_{\Psi}\right)+\left(v \mid n_{\Psi}\right)\right\},
$$

where the minimizing wavefunction $\Psi^{\text {imp }}$ fulfils the self-consistent equation

$$
\begin{aligned}
& \left(\hat{\mathcal{T}}+U \hat{n}_{0 \uparrow} \hat{n}_{0 \downarrow}+\sum_{i}\left[v_{i}+\frac{\partial \bar{E}_{\mathrm{Hxc}}^{\mathrm{bath}}\left(n_{\Psi^{\mathrm{imp}}}\right)}{\partial n_{i}}\right] \hat{n}_{i}\right)\left|\Psi^{\mathrm{imp}}\right\rangle \\
& =\mathcal{E}^{\mathrm{imp}}\left|\Psi^{\mathrm{imp}}\right\rangle .
\end{aligned}
$$

Thus we obtain an exact embedding scheme where the bath is described by a site-occupation functional. Its connection with DMET will be discussed in the following. Let us first focus on the complementary bath functional. From the KS decomposition

$$
F^{\mathrm{imp}}(n)=\mathcal{T}_{\mathrm{s}}(n)+E_{\mathrm{Hxc}}^{\mathrm{imp}}(n)
$$

where $E_{\mathrm{Hxc}}^{\mathrm{imp}}(n)$ describes the repulsion on the impurity that is embedded into a non-interacting bath, we obtain with Eqs. (34) and (42),

$$
\bar{E}_{\mathrm{Hxc}}^{\mathrm{bath}}(n)=E_{\mathrm{Hxc}}(n)-E_{\mathrm{Hxc}}^{\mathrm{imp}}(n) .
$$

This expression is convenient for developing approximate functionals. For example, within the mean-field approximation, the Hxc functional for the impurity is simplified as follows

$$
E_{\mathrm{Hxc}}^{\mathrm{imp}}(n) \rightarrow \frac{U}{4} n_{0}^{2},
$$


Figure 1. Graphical representation of the AC between the non-interacting (KS), the embedded and the physical systems. Sites labelled with $\lambda U$ (in red) and $U$ (in blue) have partial and full on-site repulsion, respectively. Non-labelled sites (in green) have no on-site repulsion. Local potentials (not represented) are adjusted so that the sites occupation is constant along the AC. See text for further details.

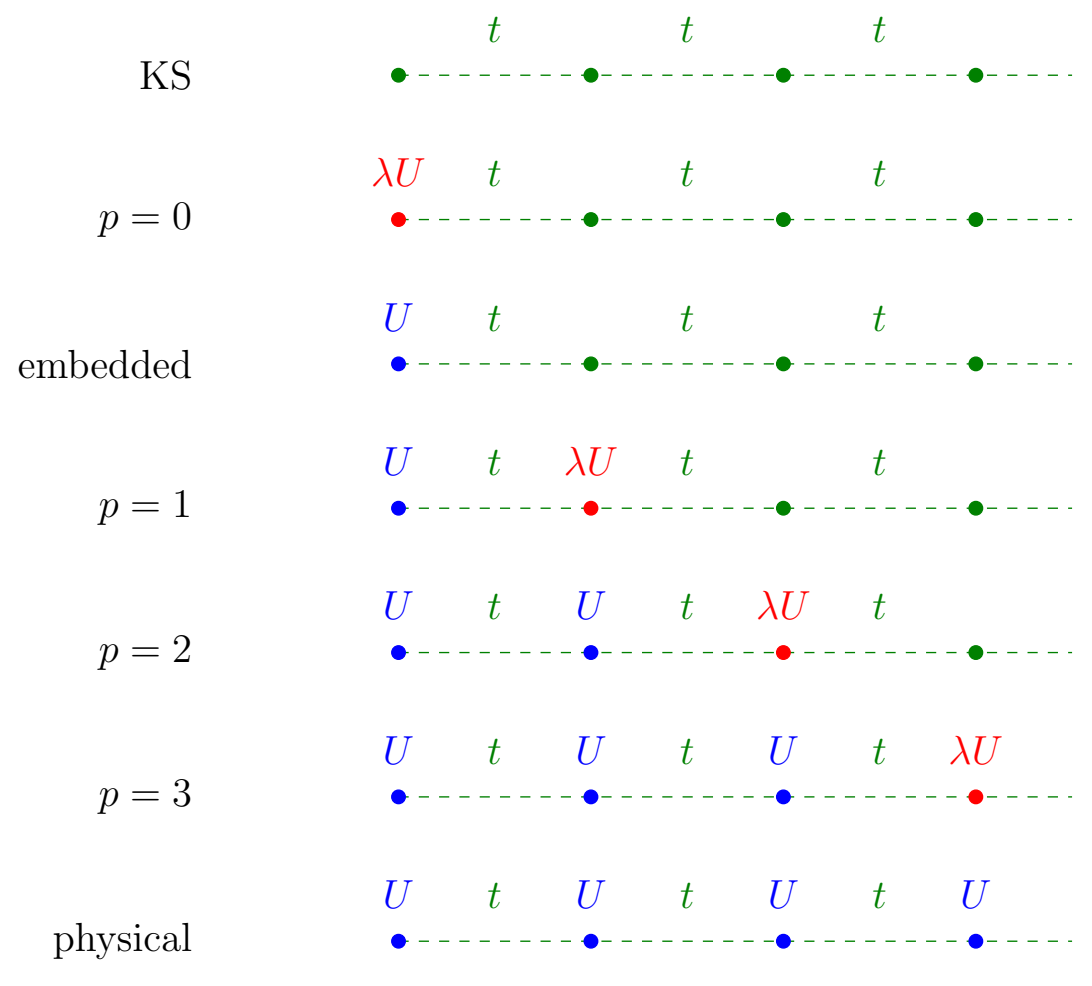

thus leading, when combined with Eq. (41), to

$$
\bar{E}_{\mathrm{Hxc}}^{\mathrm{bath}}(n) \rightarrow \frac{U}{4} \sum_{i>0} n_{i}^{2}
$$

Better approximate functionals could be developed from the local density approximation for a Luttinger liquid [34], in complete analogy with the development of short-range functionals from the uniform electron gas [37]. Work is currently in progress in this direction.

Returning to the exact theory, a more explicit expression for the complementary bath functional can be obtained within the AC formalism when considering, in analogy with Eq. (39), the following auxiliary equations

$$
\begin{aligned}
& \left(\hat{\mathcal{T}}+\hat{U}_{p-1}+\lambda U \hat{n}_{p \uparrow} \hat{n}_{p \downarrow}+\sum_{i} v_{i}^{\lambda, p} \hat{n}_{i}\right)\left|\Psi^{\lambda, p}\right\rangle \\
& =\mathcal{E}^{\lambda, p}\left|\Psi^{\lambda, p}\right\rangle, \quad p=0,1, \ldots
\end{aligned}
$$

where $\hat{U}_{p-1}=U \sum_{i=0}^{p-1} \hat{n}_{i \uparrow} \hat{n}_{i \downarrow}$ for $p \geq 1, \hat{U}_{-1}=0$, and $0 \leq \lambda \leq 1$. In such an $\mathrm{AC}$ the on-site repulsion is switched on continuously site after site. A graphical representation is given in Fig. 1. The embedded impurity system is recovered when $p=0$ and $\lambda=1$ or, equivalently, when $p=1$ and $\lambda=0$. From Eqs. (43) and (47), the site-occupation constraint $n_{\Psi^{\lambda, p}}=n$ and the Hellmann-Feynman theorem, we 
obtain

$$
\begin{aligned}
E_{\mathrm{Hxc}}^{\mathrm{imp}}(n) & =\int_{0}^{1} \mathrm{~d} \lambda \frac{\mathrm{d} \mathcal{E}^{\lambda, 0}}{\mathrm{~d} \lambda}+\left(v^{0,0}-v^{1,0} \mid n\right) \\
& =U \int_{0}^{1} \mathrm{~d} \lambda\left\langle\Psi^{\lambda, 0}\left|\hat{n}_{0 \uparrow} \hat{n}_{0 \downarrow}\right| \Psi^{\lambda, 0}\right\rangle .
\end{aligned}
$$

By introducing the multiple-impurity LL functional

$$
F_{p}^{\mathrm{imp}}(n)=\min _{\Psi \rightarrow n}\left\{\left\langle\Psi\left|\hat{\mathcal{T}}+\hat{U}_{p}\right| \Psi\right\rangle\right\}
$$

the (fully-interacting) Hxc functional becomes

$$
E_{\mathrm{Hxc}}(n)=\sum_{p \geq 0} F_{p}^{\mathrm{imp}}(n)-F_{p-1}^{\mathrm{imp}}(n)
$$

since $F_{-1}^{\mathrm{imp}}(n)=\mathcal{T}_{\mathrm{s}}(n)$, thus leading to the alternative expression (see Eq. 40 ),

$$
\begin{aligned}
E_{\mathrm{Hxc}}(n) & =\sum_{p \geq 0}\left(\int_{0}^{1} \mathrm{~d} \lambda \frac{\mathrm{d} \mathcal{E}^{\lambda, p}}{\mathrm{~d} \lambda}+\left(v^{0, p}-v^{1, p} \mid n\right)\right) \\
& =U \sum_{p \geq 0} \int_{0}^{1} \mathrm{~d} \lambda\left\langle\Psi^{\lambda, p}\left|\hat{n}_{p \uparrow} \hat{n}_{p \downarrow}\right| \Psi^{\lambda, p}\right\rangle .
\end{aligned}
$$

Combining Eqs. (48), (52) and (55) finally gives the exact expression

$$
\bar{E}_{\mathrm{Hxc}}^{\mathrm{bath}}(n)=U \sum_{p \geq 1} \int_{0}^{1} \mathrm{~d} \lambda\left\langle\Psi^{\lambda, p}\left|\hat{n}_{p \uparrow} \hat{n}_{p \downarrow}\right| \Psi^{\lambda, p}\right\rangle .
$$

If the exact complementary bath functional was known, its derivative could be computed and, by solving Eq. (46) self-consistently, we would obtain the exact embedded impurity wavefunction $\Psi^{\text {imp }}$ that has the same sites occupation $n$ as the physical system described by the Hubbard Hamiltonian in Eq. (31):

$$
n_{\Psi^{\mathrm{imp}}}=n .
$$

If $n$ is known, the exact embedding potential $v+\partial \bar{E}_{\mathrm{Hxc}}^{\mathrm{bath}}\left(n_{\Psi^{\mathrm{imp}}}\right) / \partial n$ can in fact be reached without knowing the complementary bath functional. Indeed, according to the variational principle, for any local potential $u \equiv\left\{u_{i}\right\}_{i}$, the embedded impurity wavefunction fulfils

$$
\mathcal{E}^{\mathrm{imp}}(u) \leq\left\langle\Psi^{\mathrm{imp}}\left|\hat{\mathcal{T}}+U \hat{n}_{0 \uparrow} \hat{n}_{0 \downarrow}\right| \Psi^{\mathrm{imp}}\right\rangle+\left(u \mid n_{\Psi^{\mathrm{imp}}}\right)
$$

where $\mathcal{E}^{\operatorname{imp}}(u)$ is the ground-state energy of $\hat{\mathcal{T}}+U \hat{n}_{0 \uparrow} \hat{n}_{0 \downarrow}+\sum_{i} u_{i} \hat{n}_{i}$. Thus we obtain from Eqs. (43), 46) and (57), the Legendre-Fenchel transform expression [17, 38 40

$$
F^{\mathrm{imp}}(n)=\sup _{u}\left\{\mathcal{E}^{\mathrm{imp}}(u)-(u \mid n)\right\}
$$


where $n$ is the input sites occupation and $u$ the embedding potential to be optimized. In practice approximations to $n$ could be used for example by performing a mean-field description of the physical system. In this case, the (approximate) embedding potential would enable to reproduce the mean-field occupation in both the impurity and the bath.

Thus a connection with DMET can be established (see Eq. (5) in Ref. [15]). A substantial difference though is that, in DMET, the potential to be optimized is the mean-field interaction operator $u$ that defines the mean-field approximation to the Hubbard Hamiltonian,

$$
\hat{H}^{\mathrm{mf}}(u)=\hat{\mathcal{T}}+u \sum_{i} \hat{n}_{i}
$$

and the sites occupation to be reproduced by the ground state of $\hat{H}^{\mathrm{mf}}(u)$ is the one obtained from the ground state of the DMET impurity Hamiltonian,

$$
\hat{H}^{\mathrm{DMET}}(u)=\hat{\mathcal{T}}^{\mathrm{DMET}}+U \hat{n}_{0 \uparrow} \hat{n}_{0 \downarrow}+\tilde{u} \sum_{i>0} \hat{n}_{i}
$$

where $\hat{\mathcal{T}}^{\text {DMET }}=\sum_{i>0, \sigma} v\left(\hat{a}_{0 \sigma}^{\dagger} \hat{a}_{i \sigma}+\hat{a}_{i \sigma}^{\dagger} \hat{a}_{0 \sigma}\right)$. The terms $v$ and $\tilde{u}$ are defined in Ref. [15] and depend indirectly on $u$. Note that for simplicity DMET equations are written here for a uniform external potential.

At a given iteration $I$ of a DMET calculation, the mean-field potential is set to $u_{I}$ and the impurity Hamiltonian generates the ground-state sites occupation $n_{I}^{\text {imp }}$. If it exists a local potential $u_{\mathrm{I}+1}$ such that the ground state $\Psi^{\mathrm{mf}}\left(u_{I+1}\right)$ of $\hat{H}^{\mathrm{mf}}\left(u_{I+1}\right)$ has the same sites occupation then, according to the variational principle, any trial potential $u$ fulfils

$$
\mathcal{E}^{\mathrm{mf}}(u) \leq\left\langle\Psi^{\mathrm{mf}}\left(u_{I+1}\right)|\hat{\mathcal{T}}| \Psi^{\mathrm{mf}}\left(u_{I+1}\right)\right\rangle+u\left(1 \mid n_{I}^{\mathrm{imp}}\right)
$$

where $\mathcal{E}^{\mathrm{mf}}(u)$ is the ground-state energy of $\hat{H}^{\mathrm{mf}}(u)$, thus leading to the LegendreFenchel transform expression:

$$
\mathcal{T}_{\mathrm{s}}\left(n_{I}^{\mathrm{imp}}\right)=\sup _{u}\left\{\mathcal{E}^{\mathrm{mf}}(u)-u\left(1 \mid n_{I}^{\mathrm{imp}}\right)\right\}
$$

If a maximum exists, it then corresponds to $u_{I+1}$. The updated occupation $n_{I+1}^{\text {imp }}$ is then obtained from $u_{I+1}$, thus generating a new potential until convergence is reached. In this respect, DMET can be considered as a KS Optimized Effective Potential (OEP) scheme since the mean-field Hamiltonian is in this context nothing but a non-interacting Hamiltonian. The OEP is used to model the interaction on the impurity. A formal analogy can actually be made with the range-separated KS-OEP approach proposed in Ref. [41], where the long-range interaction is described with an OEP while the short-range interaction is modeled with a density functional. The OEP is then obtained from a density constraint, exactly like in DMET.

Returning to SOET, let us finally mention that the theory can be extended to 
more impurity sites simply by using the following partitioning of the LL functional,

$$
F(n)=F_{L}^{\mathrm{imp}}(n)+\bar{E}_{\mathrm{Hxc}, L}^{\mathrm{bath}}(n),
$$

where $L \geq 0$. In this case, the AC formula for the complementary bath functional becomes

$$
\bar{E}_{\mathrm{Hxc}, L}^{\mathrm{bath}}(n)=U \sum_{p \geq L+1} \int_{0}^{1} \mathrm{~d} \lambda\left\langle\Psi^{\lambda, p}\left|\hat{n}_{p \uparrow} \hat{n}_{p \downarrow}\right| \Psi^{\lambda, p}\right\rangle .
$$

\section{Illustrative example: the two-site Hubbard model}

SOET is applied in this section to the simple two-site Hubbard model with a uniform external potential. The analytical construction of the AC is presented in Sec. 5.1. The resulting integrand expressions are then analyzed in Sec. 5.2 .

\subsection{Symmetry breaking and restoration along the AC}

Let us consider a two-electron system described by the two-site Hubbard Hamiltonian

$$
\begin{aligned}
\hat{H}= & -t \sum_{\sigma}\left(\hat{a}_{0 \sigma}^{\dagger} \hat{a}_{1 \sigma}+\hat{a}_{1 \sigma}^{\dagger} \hat{a}_{0 \sigma}\right)+U_{0} \hat{n}_{0 \uparrow} \hat{n}_{0 \downarrow} \\
& +U_{1} \hat{n}_{1 \uparrow} \hat{n}_{1 \downarrow}+v_{0} \hat{n}_{0}+v_{1} \hat{n}_{1} .
\end{aligned}
$$

Since we are interested here in the singlet ground state only, the matrix representation of the Hamiltonian can be reduced to the basis of the "doubly-occupied site" states $\left|D_{i}\right\rangle=\hat{a}_{i \uparrow}^{\dagger} \hat{a}_{i \downarrow}^{\dagger}|\operatorname{vac}\rangle$ with $i=0$ or 1 , and $|S\rangle=1 / \sqrt{2}\left(\hat{a}_{0 \uparrow}^{\dagger} \hat{a}_{1 \downarrow}^{\dagger}-\hat{a}_{0 \downarrow}^{\dagger} \hat{a}_{1 \uparrow}^{\dagger}\right)|\operatorname{vac}\rangle$ that corresponds to singly-occupied sites, thus leading to

$$
[\hat{H}]=\left[\begin{array}{ccc}
U_{0}+v_{0}-v_{1} & 0 & -\sqrt{2} t \\
0 & U_{1}+v_{1}-v_{0}-\sqrt{2} t \\
-\sqrt{2} t & -\sqrt{2} t & 0
\end{array}\right]+\left(v_{0}+v_{1}\right)
$$

For simplicity we choose for the physical Hamiltonian $v_{0}=v_{1}=0$ and $U_{0}=U_{1}=$ $U$. Consequently the site occupation is uniform:

$$
n_{0}=n_{1}=1 \text {. }
$$

We obtain by diagonalization the well-known expressions for the ground-state energy

$$
E(U)=\frac{1}{2}\left(U-\sqrt{U^{2}+16 t^{2}}\right)
$$

and the corresponding wavefunction

$$
|\Psi(U)\rangle=d(U)\left(\left|D_{0}\right\rangle+\left|D_{1}\right\rangle\right)+\sqrt{1-2 d^{2}(U)}|S\rangle
$$


where

$$
d^{2}(U)=\frac{E^{2}(U)}{8 t^{2}+2 E^{2}(U)}=\frac{E(U)}{4 E(U)-2 U}
$$

since $E^{2}(U)-U E(U)-4 t^{2}=0$, thus leading to the more explicit expression

$$
d^{2}(U)=\frac{1}{4}\left(1-\frac{1}{\sqrt{1+\frac{16}{(U / t)^{2}}}}\right) .
$$

Note the Hellmann-Feynman theorem,

$$
d^{2}(U)=\frac{1}{2} \frac{\mathrm{d} E(U)}{\mathrm{d} U}
$$

that will be used in the following. The double occupancy is the same for both sites and equal to

$$
\left\langle\Psi(U)\left|\hat{n}_{0 \uparrow} \hat{n}_{0 \downarrow}\right| \Psi(U)\right\rangle=\left\langle\Psi(U)\left|\hat{n}_{1 \uparrow} \hat{n}_{1 \downarrow}\right| \Psi(U)\right\rangle=d^{2}(U) .
$$

Along the AC described in Eq. (51) and Fig. 1, symmetry in the on-site repulsions is broken in the auxiliary Hamiltonian operator. This is clear for the embbeded impurity where $U_{0}=U$ and $U_{1}=0$. Nevertheless, symmetry can be restored in the Hamiltonian matrix simply by adjusting the local potential components $v_{0}$ and $v_{1}$ such that

$$
U_{0}+v_{0}-v_{1}=U_{1}+v_{1}-v_{0}
$$

thus leading to

$$
v_{1}-v_{0}=\frac{U_{0}-U_{1}}{2}
$$

The latter condition defines uniquely (up to a constant) the local embedding potential that gives a uniform site occupation. In this case the effective on-site repulsion is the same on each site and is simply expressed as

$$
U_{\text {eff }}=\frac{U_{0}+U_{1}}{2}
$$

Therefore the AC can be constructed analytically as follows for the impurity ( $p=$ $0)$,

$$
U_{0}=\lambda U, U_{1}=0 \rightarrow v_{0}^{\lambda, 0}=0, v_{1}^{\lambda, 0}=\lambda U / 2, U_{\text {eff }}=\lambda U / 2,
$$

and for the bath $(p=1)$,

$$
U_{0}=U, U_{1}=\lambda U \rightarrow v_{0}^{\lambda, 1}=0, v_{1}^{\lambda, 1}=(1-\lambda) U / 2, U_{\text {eff }}=(1+\lambda) U / 2 .
$$

Consequently the wavefunctions along the $\mathrm{AC}$ for the bath and the impurity are

$$
\Psi^{\lambda, 0}=\Psi(\lambda U / 2)
$$


and

$$
\Psi^{\lambda, 1}=\Psi((1+\lambda) U / 2)
$$

respectively. The corresponding double occupancies, that are nothing but Hxc integrands per unit of $U$ for the impurity and the bath (see Eqs. (52) and (56)), respectively, can then be expressed, according to Eqs. (72) and (74), as

$$
\left\langle\Psi^{\lambda, 0}\left|\hat{n}_{0 \uparrow} \hat{n}_{0 \downarrow}\right| \Psi^{\lambda, 0}\right\rangle=\frac{1}{4}\left(1-\frac{1}{\sqrt{1+\frac{64}{\lambda^{2}(U / t)^{2}}}}\right),
$$

and

$$
\left\langle\Psi^{\lambda, 1}\left|\hat{n}_{1 \uparrow} \hat{n}_{1 \downarrow}\right| \Psi^{\lambda, 1}\right\rangle=\frac{1}{4}\left(1-\frac{1}{\sqrt{1+\frac{64}{(1+\lambda)^{2}(U / t)^{2}}}}\right)
$$

Note that the embedded impurity wavefunction is obtained when $\lambda=1$ along the $\mathrm{AC}$ for the impurity, and $\lambda=0$ along the $\mathrm{AC}$ for the bath:

$$
\Psi^{\mathrm{imp}}=\Psi^{1,0}=\Psi^{0,1}=\Psi(U / 2)
$$

Since, according to Eqs. (67) and (78),

$$
\left\langle\Psi^{\mathrm{imp}}\left|\hat{\mathcal{T}}+U \hat{n}_{0 \uparrow} \hat{n}_{0 \downarrow}+(U / 2) \hat{n}_{1}\right| \Psi^{\mathrm{imp}}\right\rangle=E(U / 2)+U / 2
$$

it comes from the site occupation constraint in Eq. (68) that

$$
\left\langle\Psi^{\mathrm{imp}}\left|\hat{\mathcal{T}}+U \hat{n}_{0 \uparrow} \hat{n}_{0 \downarrow}\right| \Psi^{\mathrm{imp}}\right\rangle=E(U / 2) .
$$

Moreover, according to Eqs. (73) and (81), the double occupancy can be rewritten along the $\mathrm{AC}$ for the bath as

$$
\left\langle\Psi^{\lambda, 1}\left|\hat{n}_{1 \uparrow} \hat{n}_{1 \downarrow}\right| \Psi^{\lambda, 1}\right\rangle=\frac{1}{U} \frac{\mathrm{d} E((1+\lambda) U / 2))}{\mathrm{d} \lambda},
$$

thus giving with Eq. (56),

$$
\bar{E}_{\mathrm{Hxc}}^{\mathrm{bath}}(n)=E(U)-E(U / 2),
$$

or, more explicitly,

$$
\bar{E}_{\mathrm{Hxc}}^{\mathrm{bath}}(n) / U=\frac{1}{4}-\frac{3 / 4}{\sqrt{1+\frac{64}{(U / t)^{2}}}+2 \sqrt{1+\frac{16}{(U / t)^{2}}}} .
$$


As expected, the physical energy $E(U)$ is recovered when adding contributions from the impurity and the bath:

$$
E(U)=\left\langle\Psi^{\mathrm{imp}}\left|\hat{\mathcal{T}}+U \hat{n}_{0 \uparrow} \hat{n}_{0 \downarrow}\right| \Psi^{\mathrm{imp}}\right\rangle+\bar{E}_{\mathrm{Hxc}}^{\mathrm{bath}}(n) .
$$

Let us finally mention that, in the conventional AC (see Eq. $(39 p)$, the on-site repulsion is scaled on both the impurity and the bath sites. In this case the interaction on the impurity is not separated from the repulsions in the bath. The corresponding $\mathrm{AC}$ will therefore be defined as

$$
U_{0}=U_{1}=\lambda U \rightarrow v_{0}^{\lambda}=v_{1}^{\lambda}=0, U_{\text {eff }}=\lambda U
$$

and

$$
\Psi^{\lambda}=\Psi(\lambda U)
$$

As a result the $\mathrm{AC}$ integrand can be expressed as

$$
\left\langle\Psi^{\lambda}|\hat{U}| \Psi^{\lambda}\right\rangle=2 U d^{2}(\lambda U)
$$

thus leading to the explicit expression

$$
\frac{\left\langle\Psi^{\lambda}|\hat{U}| \Psi^{\lambda}\right\rangle}{U}=\frac{1}{2}\left(1-\frac{1}{\sqrt{1+\frac{16}{\lambda^{2}(U / t)^{2}}}}\right)
$$

Note that, according to Eq. (73), the Hxc integrand can also be rewritten as

$$
\left\langle\Psi^{\lambda}|\hat{U}| \Psi^{\lambda}\right\rangle=\frac{\mathrm{d} E(\lambda U)}{\mathrm{d} \lambda}
$$

Finally by rewriting the double occupancy along the AC for the impurity (see Eq. (82)) as follows,

$$
\left\langle\Psi^{\lambda, 0}\left|\hat{n}_{0 \uparrow} \hat{n}_{0 \downarrow}\right| \Psi^{\lambda, 0}\right\rangle=\frac{1}{U} \frac{\mathrm{d} E(\lambda U / 2)}{\mathrm{d} \lambda},
$$

we see from Eqs. (40), (55), (87) and (95) that, as expected, both the conventional Hxc integrand and the sum of bath and impurity integrands lead to the same Hxc energy after integration over $[0,1]$ :

$$
E_{\mathrm{Hxc}}(n)=E(U)-E(0)=(E(U / 2)-E(0))+(E(U)-E(U / 2)),
$$

or, more explicitly,

$$
E_{\mathrm{Hxc}}(n) / U=\frac{1}{2}\left(1+\frac{4}{U / t}-\sqrt{1+\frac{16}{(U / t)^{2}}}\right) .
$$


Figure 2. Double occupancy along the AC for the impurity plotted with respect to the interaction (top panel) and correlation (bottom panel) strenghts. See text for further details.
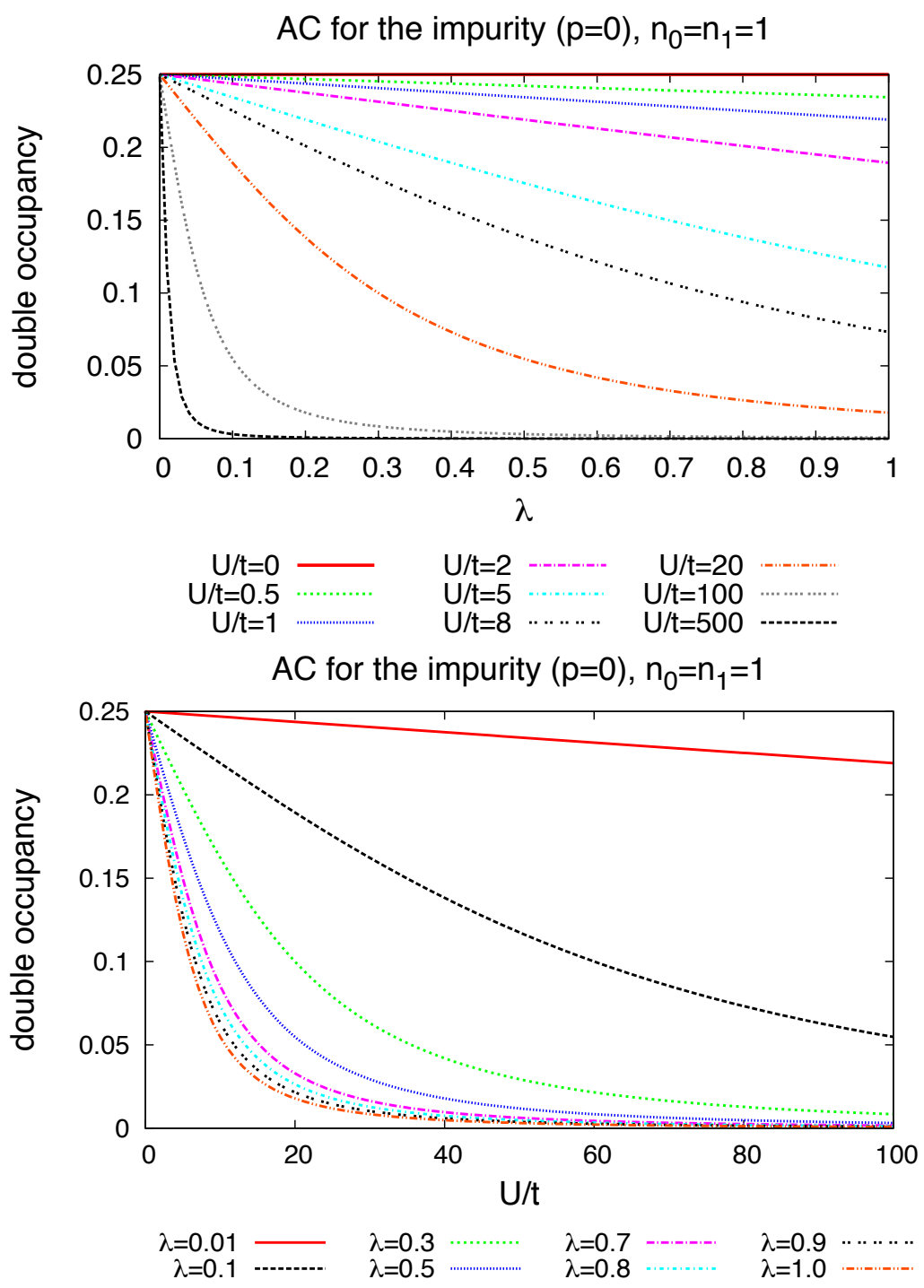

\subsection{Results and discussion}

Double occupancies along the AC for the impurity and the bath (see Eqs. (82) and (83)), that can be identified as Hxc integrands per unit of $U$, have been plotted for various correlation regimes. Results are shown in Figs. 2 and 3. For analysis purposes, comparison is made between the sum of these two integrands and the conventional Hxc integrand in Eq. (94). Results are shown in Fig. 4 .

Let us first focus on the impurity. As expected from accurate quantum chemical calculations of the $\mathrm{AC}$ for the $\mathrm{H}_{2}$ molecule at equilibrium [42, the integrand varies linearly with the interaction strength $\lambda$ in the weakly correlated regime $(U / t<<1)$. The integrand gains curvature as $U / t$ increases. In the strongly correlated regime $(U / t>>1)$, the double occupancy of the impurity becomes zero for large interaction strengths (see the bottom panel in Fig. 2p. It only varies in 
Figure 3. Double occupancy along the AC for the bath plotted with respect to the interaction (top panel) and correlation (bottom panel) strenghts. See text for further details.
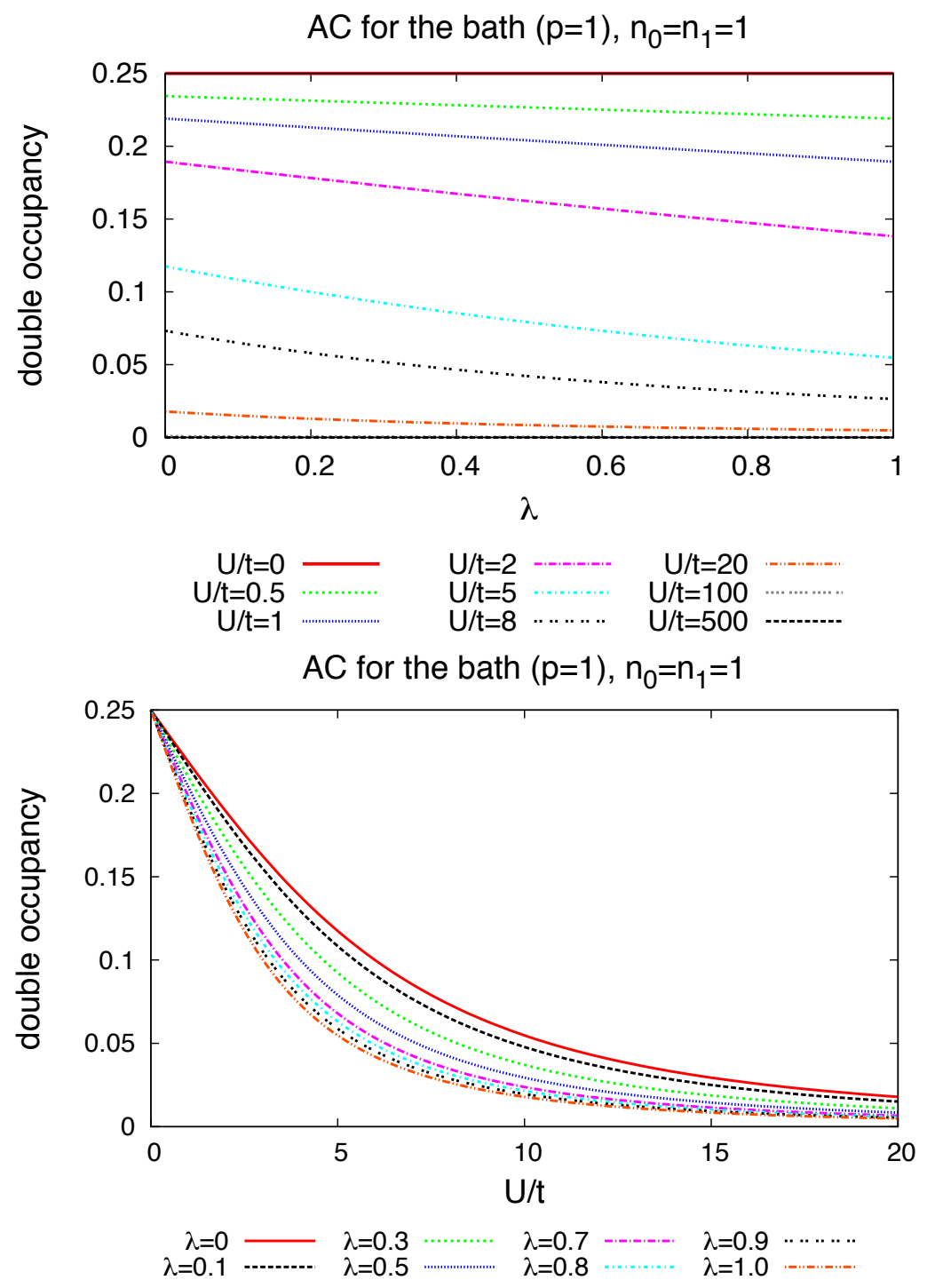

the vicinity of the non-interacting case $(\lambda=0)$ with a large negative slope. This pattern was found by Teale et al. for the stretched $\mathrm{H}_{2}$ molecule when computing the AC at the Full Configuration Interaction (FCI) level in a large basis set (see Fig. 5 (a) in Ref. [42]). One should mention that, along the AC for the impurity, the on-site repulsion in the bath is set to zero. Comparison with Ref. [42] is then more relevant when considering the conventional AC integrand for the two sites. As shown in Fig. 4, all correlation regimes observed numerically for $\mathrm{H}_{2}$ along the $\mathrm{AC}$ and the bond breaking coordinate are qualitatively well reproduced by applying SOFT to the two-site Hubbard Hamiltonian.

Turning to the AC for the bath, we first notice in the top panel of Fig. 3 that the double occupancy of the bath remains essentially linear in $\lambda$ for all correlation regimes, in contrast to the $\mathrm{AC}$ for the impurity. This is due to the fact that the on-site repulsion is already switched on for the impurity. Scaling is only used for 
Figure 4. Hxc integrands per unit of $U$ plotted for the bath, bath+impurity and the two sites. Various correlation regimes are considered: $U / t=0.05$ (red), 0.2 (green), 1 (grey), 10 (black), 30 (blue) and 300 (purple). See text for further details.

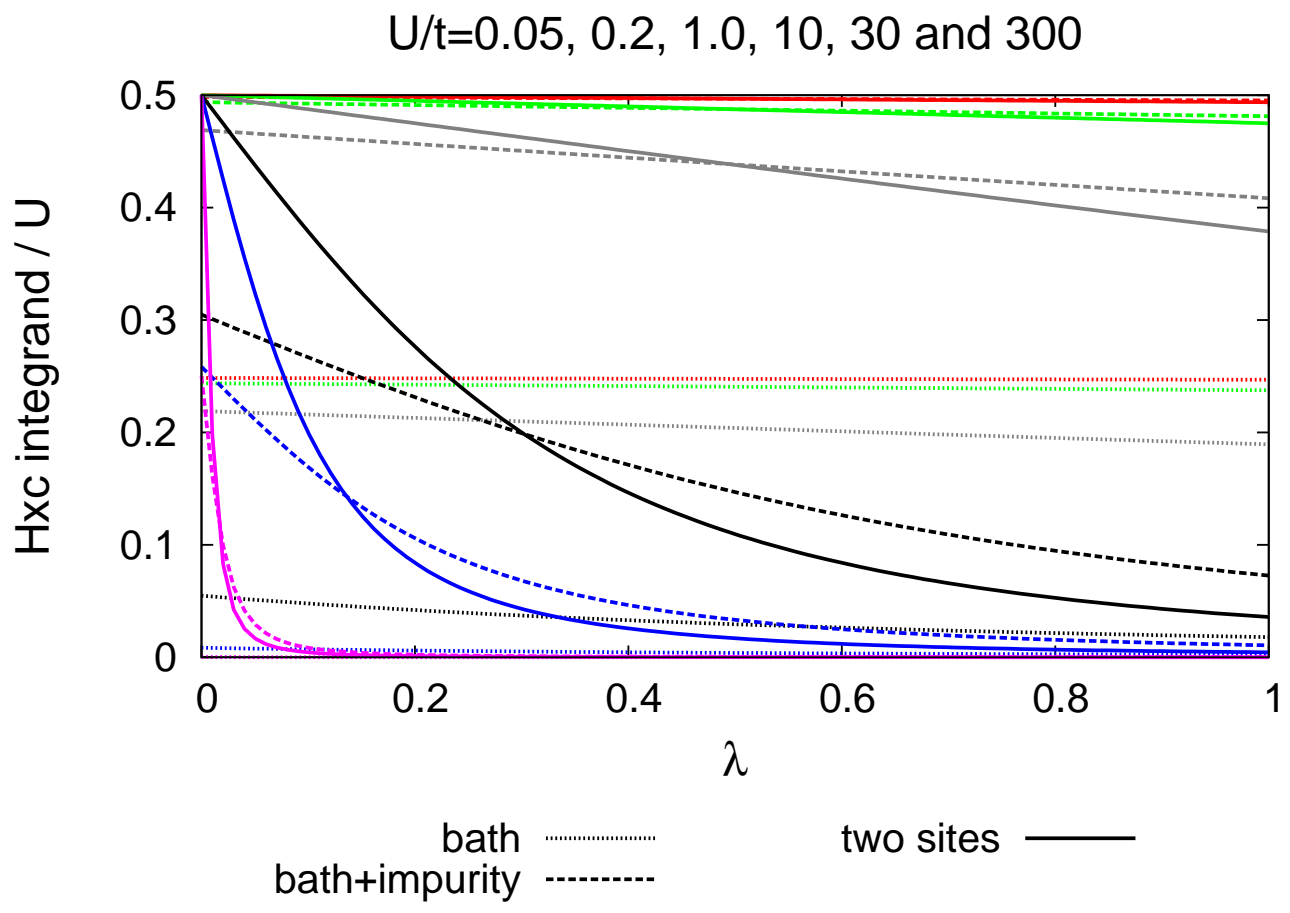

the repulsion on the bath site so that the embedded impurity $(\lambda=0)$ can be connected with the physical system $(\lambda=1)$. As expected, the two systems exhibit different double occupancies even though they have the same sites occupation (see the bottom panel in Fig. 3). Variations with the correlation strength are consistent with those obtained for the 1D-Hubbard model (see case $\langle n>=1$ in Fig. 2 of Ref. [15]).

Let us finally discuss the separation of interactions between the impurity and the bath. As mentioned previously and illustrated in Fig. 4, both the conventional and bath+impurity AC integrands give the same total Hxc energy after integration over $[0,1]$ but they of course differ along the AC. In the weakly correlated regime, both the bath and the impurity contribute significantly to the Hxc energy. Note that, in practical calculations, we want to describe the embedded impurity only, meaning that the contributions of the bath to the Hxc energy will be described by a site-occupation functional. It becomes clear from this simple example that the latter can contribute significantly and that approximations beyong the mean field (which is exact only when $U / t=0$ ) will be needed. Returning to the AC, in the strongly correlated regime, the contribution of the bath becomes negligible for all interaction strengths. Note that, for $\lambda=0$, the impurity and conventional Hxc integrands are equal to $1 / 4$ and $1 / 2$, respectively. Since both are non-zero only for small interaction strengths and behave similarly (with a large negative slope), 
they both integrate to the same Hxc energy simply because the effective on-site repulsion along the $\mathrm{AC}$ is smaller for the impurity alone $\left(U_{\text {eff }}=\lambda U / 2\right)$ than for the two sites $\left(U_{\text {eff }}=\lambda U\right)$.

\section{Applying SOFT to quantum chemistry}

We propose in this section to apply SOFT to quantum chemical Hamiltonians. The formulation of a KS scheme in this context is discussed in Sec. 6.1. We then show in Sec. 6.2 that SOET can lead to an alternative CASDFT method.

\subsection{KS-SOFT for a quantum chemical Hamiltonian}

In order to adapt SOFT to quantum chemistry, let us first consider the orthonormal basis of molecular orbitals $\left\{\phi_{p}(\mathbf{r})\right\}_{p}$ obtained for non-interacting electrons, so that

$$
\hat{h} \phi_{p}=h_{p p} \phi_{p}
$$

with $\hat{h}=-\frac{1}{2} \nabla_{\mathbf{r}}^{2}+v_{\text {ne }}(\mathbf{r})$. This is obviously not a good starting point for conventional quantum chemical calculations but it is convenient for deriving a KS-SOFT scheme in this context. As explained further in the following, the theory can in principle be adapted to different choices of orbitals. Using Eq. (99) leads to the following second-quantized expression for the molecular Hamiltonian $\hat{H}=\hat{T}+\hat{W}_{\text {ee }}+\hat{V}_{\text {ne }}$,

$$
\hat{H}=\sum_{p} h_{p p} \hat{n}_{p}+\hat{U}^{\mathrm{HF}}+\hat{\mathcal{W}}_{\mathrm{ee}}
$$

where $\hat{\mathcal{W}}_{\text {ee }}=\hat{W}_{\text {ee }}-\hat{U}^{\mathrm{HF}}$ denotes the fluctuation potential. The Hartree-Fock (HF) potential operator can be decomposed as follows,

$$
\begin{aligned}
\hat{U}^{\mathrm{HF}} & =\sum_{p} u_{p p} \hat{n}_{p}+\hat{\mathcal{T}}^{\mathrm{HF}}, \\
\hat{\mathcal{T}}^{\mathrm{HF}} & =\sum_{p \neq q, \sigma} u_{p q} \hat{a}_{p \sigma}^{\dagger} \hat{a}_{q \sigma}, \\
u_{p q} & =\sum_{r \in \mathrm{occ}} 2\langle p r \mid q r\rangle-\langle p r \mid r q\rangle,
\end{aligned}
$$

where $\langle p q \mid r s\rangle$ denotes a regular two-electron integral. The first term on the righthand side of Eq. (101) is local in the orbital space while the second one is nonlocal and thus enables hopping between occupied and unoccupied orbitals, hence the notation $\hat{\mathcal{T}}^{\mathrm{HF}}$ in analogy with the kinetic energy operator in the Hubbard Hamiltonian. Therefore the molecular Hamiltonian becomes

$$
\hat{H}=\hat{\mathcal{T}}^{\mathrm{HF}}+\hat{\mathcal{W}}_{\mathrm{ee}}+\sum_{p} \varepsilon_{p} \hat{n}_{p}
$$

with $\varepsilon_{p}=h_{p p}+u_{p p}$. This expression enables a direct comparison with the Hubbard Hamiltonian in Eq. (31): the sites are now molecular orbitals and their energies $\varepsilon \equiv\left\{\varepsilon_{p}\right\}_{p}$ play the role of the external potential. 
Following Sec. 4.1, we introduce the LL functional

$$
F(n)=\min _{\Psi \rightarrow n}\left\{\left\langle\Psi\left|\hat{\mathcal{T}}^{\mathrm{HF}}+\hat{\mathcal{W}}_{\mathrm{ee}}\right| \Psi\right\rangle\right\}
$$

so that the exact ground-state energy, that is the FCI energy in this context, can be written as

$$
E(\varepsilon)=\min _{n}\{F(n)+(\varepsilon \mid n)\}
$$

Let us consider the KS partitioning,

$$
F(n)=\mathcal{T}_{\mathrm{s}}^{\mathrm{HF}}(n)+E_{\mathrm{c}}(n)
$$

where $\mathcal{T}_{\mathrm{s}}^{\mathrm{HF}}(n)=\min _{\Psi \rightarrow n}\left\{\left\langle\Psi\left|\hat{\mathcal{T}}^{\mathrm{HF}}\right| \Psi\right\rangle\right\}$ and, in contrast to Eq. 34 , no Hartree and exchange energy contributions have been introduced. Indeed, the fluctuation potential induces correlation effects only since, when written with normal ordering [43], it generates double excitations only. Consequently, within KS-SOFT, the FCI energy is obtained variationally as follows,

$$
E(\varepsilon)=\min _{\Psi}\left\{\left\langle\Psi\left|\hat{\mathcal{T}}^{\mathrm{HF}}\right| \Psi\right\rangle+E_{\mathrm{c}}\left(n_{\Psi}\right)+\left(\varepsilon \mid n_{\Psi}\right)\right\}
$$

where the minimizing wavefunction $\Psi^{\mathrm{KS}}$ fulfils the self-consistent equation

$$
\left(\hat{\mathcal{T}}^{\mathrm{HF}}+\sum_{p}\left[\varepsilon_{p}+\frac{\partial E_{\mathrm{c}}\left(n_{\Psi^{\mathrm{KS}}}\right)}{\partial n_{p}}\right] \hat{n}_{p}\right)\left|\Psi^{\mathrm{KS}}\right\rangle=\mathcal{E}^{\mathrm{KS}}\left|\Psi^{\mathrm{KS}}\right\rangle .
$$

Eqs. (108) and (109) should in principle enable to recover the FCI energy without treating electron correlation explicitly. Orbital rotations would therefore be sufficient. One should of course investigative potential $\varepsilon$-representability problems for example by computing Legendre-Fenchel transforms. Approximate functionals may also be developed from model Hamiltonians such as the Hubbard Hamiltonian. Let us finally note that different orbitals could be used in Eq. (109). One would need to adapt the correlation functional in order to recover the correct FCI energy. Work is in progress in these directions.

\subsection{CASSOFT approach}

Following Knizia and Chan [44, we propose in this section to apply SOET to the molecular Hamiltonian in Eq. (104). The impurity sites will be the active orbitals $(u, v, \ldots)$ whose selection is usually based on chemical intuition, while the inactive and virtual orbitals correspond to the bath. A graphical representation is given in Fig. 5. As already mentioned for SOET, the separation of correlation effects is not controlled here by a single continuous parameter like in range-separated DFT. In the spirit of a regular CASSCF calculation, it rather relies on the selection of active orbitals. This ensures that only correlation effects within the active space are described explicitly in WFT while the remaining correlation, including core correlation, is modeled by an orbital-occupation functional. Separating correlation effects in the active orbital space $\mathcal{A}$ from the remaining ones leads to the alternative 
partitioning of the LL functional,

$$
F(n)=F_{\mathcal{A}}^{\mathrm{imp}}(n)+\bar{E}_{\mathrm{c}}^{\mathrm{bath}}(n),
$$

where

$$
F_{\mathcal{A}}^{\mathrm{imp}}(n)=\min _{\Psi \rightarrow n}\left\{\left\langle\Psi\left|\hat{\mathcal{T}}^{\mathrm{HF}}+\hat{\mathcal{W}}_{\mathcal{A}}\right| \Psi\right\rangle\right\}
$$

and $\hat{\mathcal{W}}_{\mathcal{A}}$ is the reduction of the fluctuation potential operator to the active orbital space:

$$
\begin{aligned}
\hat{\mathcal{W}}_{\mathcal{A}}= & \frac{1}{2} \sum_{u, v, x, y, \sigma, \sigma^{\prime}}\langle u v \mid x y\rangle \hat{a}_{u \sigma}^{\dagger} \hat{a}_{v \sigma^{\prime}}^{\dagger} \hat{a}_{y \sigma^{\prime}} \hat{a}_{x \sigma} \\
& -\sum_{u, v} \sum_{x \in \mathrm{occ}}(2\langle u x \mid v x\rangle-\langle u x \mid x v\rangle) \hat{a}_{u \sigma}^{\dagger} \hat{a}_{v \sigma} .
\end{aligned}
$$

Note that the HF potential in the second term on the right-hand side of Eq. (112) is here calculated with the active orbitals that are doubly occupied, by analogy with Eq. (31) in Ref. [45. Combining Eqs. (106), 110) and (111) leads to the exact variational expression

$$
E(\varepsilon)=\min _{\Psi}\left\{\left\langle\Psi\left|\hat{\mathcal{T}}^{\mathrm{HF}}+\hat{\mathcal{W}}_{\mathcal{A}}\right| \Psi\right\rangle+\bar{E}_{\mathrm{c}}^{\mathrm{bath}}\left(n_{\Psi}\right)+\left(\varepsilon \mid n_{\Psi}\right)\right\}
$$

where the minimizing wavefunction $\Psi_{\mathcal{A}}^{\mathrm{imp}}$ fulfils the self-consistent equation

$$
\left(\hat{\mathcal{T}}^{\mathrm{HF}}+\hat{\mathcal{W}}_{\mathcal{A}}+\sum_{p}\left[\varepsilon_{p}+\frac{\partial \bar{E}_{\mathrm{c}}^{\mathrm{bath}}\left(n_{\Psi_{\mathcal{A}}^{\mathrm{imp}}}\right)}{\partial n_{p}}\right] \hat{n}_{p}\right)\left|\Psi_{\mathcal{A}}^{\mathrm{imp}}\right\rangle=\mathcal{E}_{\mathcal{A}}^{\mathrm{imp}}\left|\Psi_{\mathcal{A}}^{\mathrm{imp}}\right\rangle
$$

The method will be referred to as CASSOFT. Interestingly the impurity Hamiltonian is very similar to the one proposed by Zgid and Chan in Ref. [45]. A major

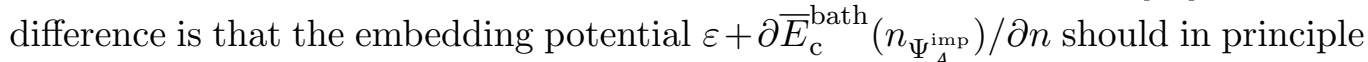
enable to recover the exact orbitals occupancy in both the bath and the impurity and, through the functional, the FCI energy. The impurity Hamiltonian can also be viewed as an embedded version of Dyall's Hamiltonian [46. A numerical validation of Eq. (114) for small molecules can be achieved when computing from the input FCI orbitals occupation $n$ the Legendre-Fenchel transform

$$
F_{\mathcal{A}}^{\mathrm{imp}}(n)=\sup _{\varepsilon}\left\{\mathcal{E}_{\mathcal{A}}^{\mathrm{imp}}(\varepsilon)-(\varepsilon \mid n)\right\}
$$

where $\mathcal{E}_{\mathcal{A}}^{\mathrm{imp}}(\varepsilon)$ is the ground-state energy of $\hat{\mathcal{T}}^{\mathrm{HF}}+\hat{\mathcal{W}}_{\mathcal{A}}+\sum_{p} \varepsilon_{p} \hat{n}_{p}$. Work is in progress in this direction.

Let us stress that, in contrast to the short-range density functional used in range-separated DFT, the complementary bath orbital-occupation functional $\bar{E}_{\mathrm{c}}^{\text {bath }}(n)$ is not a universal functional. It will a priori depend on the molecular orbital basis in which CASSOFT is formulated. It also depends on the active orbital space. Even though range-separated DFT is much simpler in that respect, the choice of an optimal range-separation parameter is, in terms of accuracy, not 
universal partly because static correlation is not a purely long-range effect. Local and semi-local approximations to the short-range density functional are usually not accurate enough for modeling strongly multi-configurational systems (see Ref. [20] and the references therein). Working in the orbital space will at least allow for a proper separation of static and dynamical correlation effects. One may consider the development of approximate functionals to be a cumbersome task in this context. A similar challenge is to some extent encountered in Natural Orbital Functional Theory (NOFT) [47]. Recent advances in the field might be useful for such developments. This is left for future work.

Returning to exact CASSOFT, an AC formula can actually be derived for the complementary bath correlation functional $\bar{E}_{\mathrm{c}}^{\text {bath }}(n)$ by introducing orbitaldependent active spaces $\mathcal{A}_{r, s}$ where the indices $r$ and $s$ refer to the lowest and highest active orbitals in energy, respectively. Let $\mathcal{A}_{r_{0}, s_{0}}$ denote the active space of interest $\mathcal{A}$. We then consider the auxiliary equations

$$
\left(\hat{\mathcal{T}}^{\mathrm{HF}}+\hat{\mathcal{W}}_{\mathcal{A}_{r, s}}+\lambda \hat{\mathcal{W}}_{\mathcal{A}_{r, s}}^{ \pm}+\sum_{p} \varepsilon_{p}^{\lambda, r s, \pm} \hat{n}_{p}\right)\left|\Psi^{\lambda, r s, \pm}\right\rangle=\mathcal{E}^{\lambda, r s, \pm}\left|\Psi^{\lambda, r s, \pm}\right\rangle
$$

where the orbitals occupation constraint $n_{\Psi^{\lambda, r s, \pm}}=n$ is fulfilled for $0 \leq \lambda \leq 1$, $0 \leq r \leq r_{0}$ and $s_{0} \leq s$. The virtual and inactive increment operators are defined as

$$
\begin{aligned}
& \hat{\mathcal{W}}_{\mathcal{A}_{r, s}}^{+}=\hat{\mathcal{W}}_{\mathcal{A}_{r, s+1}}-\hat{\mathcal{W}}_{\mathcal{A}_{r, s}}, \\
& \hat{\mathcal{W}}_{\mathcal{A}_{r, s}}^{-}=\hat{\mathcal{W}}_{\mathcal{A}_{r-1, s}}-\hat{\mathcal{W}}_{\mathcal{A}_{r, s}},
\end{aligned}
$$

respectively. The explicit expression for $\hat{\mathcal{W}}_{\mathcal{A}_{r, s}}$ is deduced from Eq. 112 . The superscripts "+" and "-" refer to the incorporation into the active space $\mathcal{A}_{r, s}$ of the virtual $s+1$ and inactive $r-1$ orbitals, respectively. A graphical representation is given in Fig. 5. The embedded active electrons are recovered along the $\mathrm{AC}$ when $r=r_{0}, s=s_{0}$ and $\lambda=0$. According to Eqs. (110) and (111), the complementary bath correlation functional can be expressed as

$$
\begin{aligned}
\bar{E}_{\mathrm{c}}^{\mathrm{bath}}(n)= & F(n)-F_{\mathcal{A}_{r_{0}, s_{0}}}^{\mathrm{imp}}(n) \\
= & \sum_{s \geq s_{0}} F_{\mathcal{A}_{0, s+1}}^{\mathrm{imp}}(n)-F_{\mathcal{A}_{0, s}}^{\mathrm{imp}}(n) \\
& -\sum_{r=1}^{r_{0}} F_{\mathcal{A}_{r, s_{0}}}^{\mathrm{imp}}(n)-F_{\mathcal{A}_{r-1, s_{0}}}^{\mathrm{imp}}(n),
\end{aligned}
$$

thus leading, by analogy with Eq. (55), to

$$
\begin{aligned}
\bar{E}_{\mathrm{c}}^{\text {bath }}(n)= & \sum_{s \geq s_{0}} \int_{0}^{1} \mathrm{~d} \lambda\left\langle\Psi^{\lambda, 0 s,+}\left|\hat{\mathcal{W}}_{\mathcal{A}_{0, s}}^{+}\right| \Psi^{\lambda, 0 s,+}\right\rangle \\
& +\sum_{r=1}^{r_{0}} \int_{0}^{1} \mathrm{~d} \lambda\left\langle\Psi^{\lambda, r s_{0},-}\left|\hat{\mathcal{W}}_{\mathcal{A}_{r, s_{0}}^{-}}\right| \Psi^{\lambda, r s_{0},-}\right\rangle .
\end{aligned}
$$

It is readily seen from Eq. (120) that the complementary bath correlation functional describes correlation effects that (i) couple inactive or active orbitals with the 
Figure 5. Graphical representation of the active spaces $\mathcal{A}_{r, s}$. Dashed blue lines represent active orbitals in $\mathcal{A}_{r_{0}, s_{0}}$. Full green lines are either inactive or virtual orbitals. See text for further details.

$$
s \geq s_{0}
$$

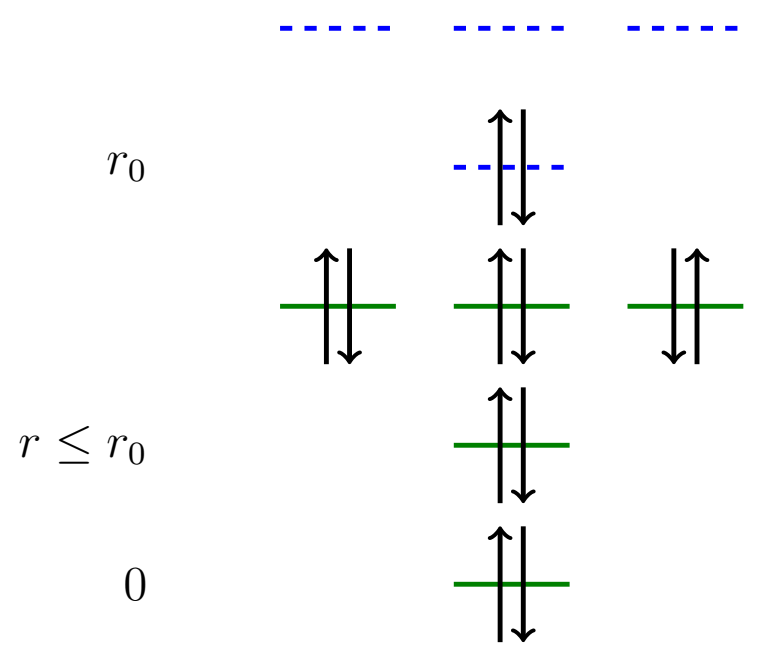

virtual orbitals (first term on the right-hand side) and that (ii) couple inactive orbitals with active orbitals (second term). The correlation effects within the active space are treated explicitly. Note that CASSOFT is in principle exact (in a given basis set) and it is free from double counting problems. The reason is that it relies on an exact separation of the LL functional in the orbital space.

\section{Conclusions and perspectives}

The exact formulation of multi-configuration density-functional theory has been discussed. The infamous double counting problem can be avoided when separating correlation effects either in the coordinate space or in the orbital space. In the latter case, orbitals occupation should be used as basic variable rather than the electron density. This approach has been applied to the Hubbard Hamiltonian, thus leading to an exact Site Occupation Embedding Theory (SOET). The connection with Density Matrix Embedding Theory (DMET) has been discussed and an adiabatic connection (AC) formula has been derived for the complementary bath functional. The AC has been constructed analytically for the simple two-site Hubbard model. The computational implementation of SOET as well as the development of approximate local bath functionals is left for future work. 
We then proposed to apply SOET to a quantum chemical Hamiltonian, thus showing that multi-configuration methods can be merged rigorously with orbitaloccupation functionals. The method is referred to as CAS Site Occupation Functional Theory (CASSOFT). In this context, impurity sites correspond to the active orbitals while inactive and virtual orbitals are the bath. An AC formalism has also been derived. The latter should be useful for developing approximate functionals. A connection with multi-reference perturbation theory may be achieved from a perturbation expansion of the AC integrand. Note also that, in regular DFT, the uniform electron gas played a crucial role in the development of electron density functionals. By analogy, model Hamiltonians such as the Hubbard Hamiltonian could be used for developing local and semi-local orbital-occupation functionals. Finally, following Knizia and Chan [15], it would be interesting to explore connections between CASSOFT and Density Matrix Renormalization Group (DMRG) methods [48]. Work is in progress in these directions.

\section{Acknowledgments}

The author would like to thank the editorial board for the kind invitation to submit a "New Views" paper to Mol. Phys. EF is grateful to Markus Reiher and Stefan Knecht for their kind invitation to give a seminar at ETH in august 2014 and their helpful comments on this work. EF thanks Masahisa Tsuchiizu, Vincent Robert, Laurent Mazouin, Andreas Savin, Lucia Reining and Bernard Amadon for stimulating discussions on strongly correlated electrons. The author finally acknowledges financial support from the PHC program Sakura 2969UK, the LABEX "Chemistry of complex systems" and the ANR (MCFUNEX project).

\section{References}

[1]K. Andersson, P.A. Malmqvist and B.O. Roos, J. Chem. Phys. 96, 1218 (1992).

[2]D. Roca-Sanjun, F. Aquilante and R. Lindh, WIREs Comput Mol Sci 2, 585 (2012).

[3] C. Angeli, R. Cimiraglia, S. Evangelisti, T. Leininger and J.P. Malrieu, J. Chem. Phys. 114, 10252 (2001).

[4]C. Angeli, R. Cimiraglia and J.P. Malrieu, J. Chem. Phys. 117, 9138 (2002).

[5]D.I. Lyakh, M. Musia, V.F. Lotrich and R.J. Bartlett, Chem. Rev. 112, 182 (2012).

[6] J. Gräffenstein and D. Cremer, Chem. Phys. Lett. 316, 569 (2000).

[7]S. Gusarov, P.Å. Malmqvist, R. Lindh and B.O. Roos, Theor. Chem. Acc. 112, 84 (2004).

[8] A.J. Pérez-Jiménez and J.M. Pérez-Jordá, Phys. Rev. A 75, 012503 (2007).

[9] M. Weimer, F. Della Sala and A. Görling, J. Chem. Phys. 128 (14), 144109 (2008).

[10] Y. Kurzweil, K.V. Lawler and M. Head-Gordon, Mol. Phys. 107, 2103 (2009).

[11]A. Savin, in Recent Developments and Applications of Modern Density Functional Theory (Elsevier, Amsterdam, 1996), p. 327.

[12] A. Savin, Int. J. Quantum Chem. S22, 59 (1988).

[13]C. Gutlé and A. Savin, Phys. Rev. A 75, 032519 (2007).

[14]K. Capelle and V.L. Campo Jr., Phys. Rep. 528, 91 (2013).

[15] G. Knizia and G.K.L. Chan, Phys. Rev. Lett. 109, 186404 (2012).

[16]T. Helgaker, P. Jørgensen and J. Olsen, in Molecular Electronic-Structure Theory (Wiley, Chichester, 2004), pp. 598-647.

[17]E. Lieb, Int. J. Quantum Chem. 24, 243 (1983).

[18] P. Hohenberg and W. Kohn, Phys. Rev. 136, B864 (1964).

[19] W. Kohn and L.J. Sham, Phys. Rev. A 140, 1133 (1965).

[20] A. Stoyanova, A.M. Teale, J. Toulouse, T. Helgaker and E. Fromager, J. Chem. Phys. 139, 134113 (2013).

[21]K. Sharkas, A. Savin, H.J.Aa. Jensen and J. Toulouse, J. Chem. Phys. 137, 044104 (2012).

[22] E. Fromager, J. Toulouse and H.J.Aa. Jensen, J. Chem. Phys. 126, 074111 (2007).

[23]E. Fromager, F. Réal, P. Wåhlin, U. Wahlgren and H.J.Aa. Jensen, J. Chem. Phys. 131, 054107 (2009).

[24]D.C. Langreth and J.P. Perdew, Solid State Commun. 17, 1425 (1975).

[25] O. Gunnarsson and B.I. Lundqvist, Phys. Rev. B 13, 4274 (1976).

[26] O. Gunnarsson and B.I. Lundqvist, Phys. Rev. B 15, 6006 (1977).

[27]D.C. Langreth and J.P. Perdew, Phys. Rev. B 15, 2884 (1977).

[28] A. Savin, F. Colonna and R. Pollet, Int. J. Quantum Chem. 93, 166 (2003).

[29]W. Yang, J. Chem. Phys. 109, 10107 (1998). 
[30] J. Toulouse, F. Colonna and A. Savin, Phys. Rev. A 70, 062505 (2004).

31] M.J.S. Dewar and J. Kelemen, J. Chem. Educ. 48, 494 (1971).

[32]P. Gori-Giorgi and A. Savin, Int. J. Quantum Chem. 109, 1950 (2009).

[33] O. Gunnarsson and K. Schönhammer, Phys. Rev. Lett. 56, 1968 (1986).

[34] N.A. Lima, M.F. Silva, L.N. Oliveira and K. Capelle, Phys. Rev. Lett. 90, 146402 (2003).

[35]A. Georges, G. Kotliar, W. Krauth and M.J. Rozenberg, Rev. Mod. Phys. 68, 13 (1996).

[36] G. Kotliar, S.Y. Savrasov, K. Haule, V.S. Oudovenko, O. Parcollet and C.A. Marianetti, Rev. Mod. Phys. 78, 865 (2006).

[37] J. Toulouse, A. Savin and H.J. Flad, Int. J. Quantum Chem. 100, 1047 (2004).

[38] H. Eschrig, The Fundamentals of Density Functional Theory, 2nd ed. (Eagle, Leipzig, 2003; Edition am Gutenbergplatz), Edition am Gutenbergplatz.

[39]W. Kutzelnigg, J. Mol. Structure: THEOCHEM 768, 163 (2006).

[40]R. van Leeuwen, Adv. Quantum Chem. 43, 25 (2003).

[41]E. Fromager and H.J. Aa. Jensen, Phys. Rev. A 78, 022504 (2008).

[42] A.M. Teale, S. Coriani and T. Helgaker, J. Chem. Phys. 133, 164112 (2010).

[43]I. Lindgren and J. Morrison, in Atomic Many-Body Theory (Springer-Verlag, Berlin, 1982), Springer Series in Chemical Physics, Vol. 13.

[44] G. Knizia and G.K.L. Chan, J. Chem. Theory Comput. 9, 1428 (2013).

[45]D. Zgid and G.K.L. Chan, J. Chem. Phys. 134, 094115 (2011).

[46] K.G. Dyall, J. Chem. Phys. 102, 4909 (1995).

[47]M. Piris and J.M. Ugalde, Int. J. Quantum Chem. 114, 1169 (2014).

[48] S.R. White, Phys. Rev. Lett. 69, 2863 (1992). 